\title{
Significance of localized soil resistivity in designing a grounding system
}

\begin{abstract}
The value of soil resistivity is an essential pre-requisite in designing an electrical grounding system. The current practice, as advocated even by national/international standards involves taking the average soil resistivity of the site as the parameter to be considered in designing the grounding system. This study shows that average soil resistivity may lead to significant overestimation or underestimation in the designing process as the localized soil resistivity may significantly differ from the average soil resistivity of the entire site. At least $14 \%$ difference between average and localized soil resistivity were obtained base on measurements done on five locations. Therefore, it is suggested that electrical engineers who design grounding systems should first identify the locations where the components of the grounding systems will be installed. Then, the localized soil resistivity at that particular point should be measured and the sizing and geometry of the grounding electrode should be designed accordingly.
\end{abstract}

